\title{
Aripiprazole for the treatment of tic disorders in children: a systematic review and meta-analysis
}

\author{
Chun-Song Yang ${ }^{1,2 \dagger}$, Hong Huang ${ }^{1 \dagger}$, Ling-Li Zhang ${ }^{1,4^{*}}$, Cai-Rong Zhu² and Qin Guo ${ }^{3}$
}

\begin{abstract}
Background: Tic disorders (TDs) are common neuropsychiatric disorders in children. Typical antipsychotics, such as haloperidol and pimozide have been prescribed to control tic symptoms as first-line agents. However, adverse effects have led to the use of newer atypical antipsychotics. Aripiprazole is one of alternatives. The aim of this study was to evaluate the efficacy and safety of aripiprazole for children with TDs.

Methods: Randomized controlled trials (RCTs), quasi-RCTs and control studies evaluating aripiprazole for children with tic disorders were identified from PubMed, Embase, Cochrane library, Cochrane Central, four Chinese database and relevant reference lists. Quality assessment referred to the Cochrane Handbook for Systematic Reviews of Interventions.
\end{abstract}

Results: Twelve studies involving 935 participants were included. The general quality of included studies was poor. Only one study used placebo as a control and others used positive drug controls. Participants were aged between 4 and 18 years. The period of treatment ranged from 8 to 12 weeks. Seven studies ( $N=600$ patients) used the YGTSS scale as the outcome measurement, and there was no significant difference in reduction of the total YGTSS score between the aripiprazole and positive control groups $(\mathrm{MD}=-0.48,95 \% \mathrm{Cl}$ $\left.[-6.22,5.26], P=0.87, I^{2}=87 \%\right)$. Meta-analysis of four of the studies ( $N=285$ patients) that compared aripiprazole with haloperidol showed that there was no significant difference in reduction of the total YGTSS score ( $M D=2.50$, $\left.95 \% \mathrm{Cl}[-6.93,11.92], P=0.60, \mathrm{I}^{2}=88 \%\right)$. Meta-analysis of two studies ( $N=255$ patients) that compared aripiprazole with tiapride showed that there was no significant difference in reduction of the total YGTSS score (MD $=-3.15$, $\left.95 \% \mathrm{Cl}[-11.38,5.09], P=0.45, I^{2}=86 \%\right)$. Adverse events (AEs) were reported in 11 studies. Drowsiness $(5.1 \%-58.1 \%)$, increased appetite (3.2 \%-25.8 \%), nausea (2 \%-18.8 \%) and headache (2\%-16.1\%) were common AEs.

Conclusion: In conclusion, aripiprazole appears to be a promising therapy for children with TDs. Further wellconducted RCTs are required to confirm this issue.

Keywords: Aripiprazole, Tic disorders, Children, Systematic review

\section{Background}

Tic disorders (TDs) are common neuropsychiatric disorders in children. These disorders are characterized by sudden, fast, repetitive, non-rhythmic, and stereotyped motor movements and/or phonic production [1]. TDs are often classified as transient tic disorder (TTD), chronic tic disorder

\footnotetext{
* Correspondence: zhlingli@sina.com

${ }^{\dagger}$ Equal contributors

'Department of Pharmacy, Evidence-based Pharmacy Center, West China second hospital, Key Laboratory of Birth Defects and Related Diseases of Women and Children, Sichuan University, Chengdu, China

${ }^{4}$ West China Second University Hospital, Sichuan University, No.20,Third Section, Renmin NanLu, Chengdu, Sichuan 610041, People's Republic of China

Full list of author information is available at the end of the article
}

(CTD), and Tourette syndrome (TS). Symptoms of common comorbidities of TDs (i.e., attention-deficit hyperactivity disorder [ADHD], obsessive-compulsive disorder, oppositional defiant disorder, and other mood disorders) often co-exist [2]. A meta-analysis of the prevalence of TDs showed that the prevalence of TS is $0.77 \%$ (95\% confidence interval [CI]: $0.39-1.51)$, TTD is the most common TD, with a prevalence of $2.99 \%$ (95 \% CI: 1.60 5.61), and CTD has a prevalence of 1.61 \% (95 \% CI: 0.922.83) [3].

Currently, pharmacotherapy is the main management for motor/vocal tics and comorbidity symptoms. Typical antipsychotics, such as haloperidol and pimozide have

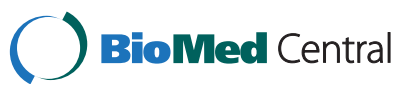

(c) 2015 Yang et al.; licensee BioMed Central. This is an Open Access article distributed under the terms of the Creative Commons Attribution License (http://creativecommons.org/licenses/by/4.0), which permits unrestricted use, distribution, and reproduction in any medium, provided the original work is properly credited. The Creative Commons Public Domain Dedication waiver (http://creativecommons.org/publicdomain/zero/1.0/) applies to the data made available in this article, unless otherwise stated. 
been prescribed to control tic symptoms as first-line agents [4]. However, adverse effects, such as acute dystonic reactions, akathisia, tardive dyskinesia, extrapyramidal syndrome, and prolonged QTc, are problematic and have led to the use of newer atypical antipsychotics [5]. Among the atypical antipsychotics, the use of risperidone and aripiprazole for the treatment of TS has been described in randomized controlled trials (RCTs) and case series studies [6-12]. In 2013, a 14-week prospective open-label study [11] evaluated the efficacy of aripiprazole for TDs in patients who were aged between 4 and 18 years, including 81 children. This previous study showed that the mean reduction in the motor tic score was $51.0 \%$, that in the vocal tic score was $67.1 \%$, and that in the total Yale Global Tic Severity Scale (YGTSS) score was $70 \%$ after treatment. The authors concluded that aripiprazole is effective for the short-term treatment of TD, especially vocal tics, with few adverse effects. In 2012, another 12-week open-label study [12] assessed the use of aripiprazole in a consecutive group of 28 patients with the primary diagnosis of TS and co-morbid ADHD, a combined subtype. This previous study showed that the YGTSS score and the ADHD rating scale IV significantly improved $(p<0.001)$ after treatment. With regard to the YGTSS, there was a reduction of $42.5 \%$ in motor tics, $47.9 \%$ in vocal tics, and $32.3 \%$ in the tic impairment. A total of $67.9 \%$ of patients had a reduction of at least $50 \%$ of the total YGTSS score [12]. Additionally the results of two RCTs that were recently published showed that aripiprazole is efficacious, generally tolerated, and safe for TDs $[9,10]$.

Aripiprazole has been used to treat schizophrenia and bipolar disorder for adults and adolescents for many years. The mechanisms of action may be a partial agonist on $\mathrm{D}_{2}$ and 5-hydroxytryptamine $1 \mathrm{~A}$ receptors and an antagonist on 5-hydroxytryptamine 2A receptors [13]. Aripiprazole may also have beneficial effects in patients with TDs with a lower risk of side effects than other atypical neuroleptics [14].

Ghanizadeh's study [15] systematically reviewed the efficacy and safety of aripiprazole for children with TDs. They concluded that aripiprazole is effective for treating tic disorders including TS in children and adolescents. Additionally, aripiprazole's adverse effect profile is safer than pimozide and some other antipsychotics. However, the included studies in this systematic review have several limitations, including a small sample size, an openlabel approach, being case reports or case series, and the short-term nature of the studies. Recently, two RCTs that evaluated the efficacy of aripiprazole for children with TDs were published $[9,10]$. Many studies have also been published in China. Therefore, this systematic review [15] may have missed some important information. In addition, the newer atypical antipsychotics can reduce the risk of extrapyramidal symptoms. However, atypical antipsychotics are associated with metabolic side effects, including weight gain and hyperlipidemia (an abnormally high concentration of fatty substances in the blood) [16]. Therefore, the evidence for the efficacy and safety of aripiprazole for children with TD needs to be updated.

\section{Methods}

Inclusion and exclusion criteria

Types of studies

All RCTs, quasi-RCTs, and open-label control studies comparing aripiprazole with placebo or other drug(s) used in the treatment of children with TDs were included. Trials were excluded if (1) the data for children could not be obtained (even though we attempted to contact the original study investigators), and (2) they compared different doses of drugs (i.e., the treatment group used high [or low] doses of aripiprazole and the control group used low [or high] doses).

\section{Types of participants}

Patients with the clinical diagnosis of a TD were included. The widely used definitions of TDs are in the following guidelines: (1) the Diagnostic and Statistical Manual of Mental Disorders-III (DSM-III), DSM-IV, or DSM-IVText Revision [17-19]; (2) the International Classification of Diseases-10 (ICD-10) [20]; and (3) the Chinese Classification and Diagnostic Criteria of Mental Disorders (CCMD) [21]. The age of participants was younger than 18 years.

\section{Types of interventions}

All RCTs, quasi-RCTs, and open-label control studies that administered aripiprazole used either alone or as an add-on to an approved treatment for TDs were included. Comparisons included (1) aripiprazole versus placebo only, (2) aripiprazole plus approved treatments versus placebo plus approved treatments, and (3) aripiprazole versus approved treatments (i.e., haloperidol and tiapride).

\section{Types of outcome measurements \\ Primary outcomes}

We included studies that measured outcomes using one of the following scales or methods: (1) the YGTSS [22]; (2) the Clinical Global Impression (CGI) Scale [23]; (3) the Tourette Syndrome Global Scale [18]; (4) the Tourette Syndrome Symptom List [18]; (5) the Clinical Global Impression Tic Severity Scale [19]; and (6) the Tourette Syndrome Severity Scale [23].

\section{Secondary outcomes}

The secondary outcomes included improvement of tic symptoms that were assessed by authors' self-definition and adverse events (AEs), which were measured using 
the following scales or methods: (1) the CGI Scale, Adverse Events [23], (2) the Abnormal Involuntary Movement Scale, (3) the Extrapyramidal Symptom Rating Scale, (4) weight gain, (5) abnormalities or changes on an electrocardiogram [18], and (6) other reported AEs.

\section{Search strategy}

Two reviewers (Yang and Huang) independently identified studies through searches of PubMed (1966-2014.6), EMBASE (1974-2014, Issue 6), the Cochrane Library (2014, Issue 6), Cochrane Controlled Trials databases (CENTRAL 6, 2014), the Chinese Biomedical Literature Database (CBM, 1978-2014.6), China National Knowledge Infrastructure (CNKI, 1980-2014.6), the Chinese Science and Technique Journals Database (VIP, 1989-2014.6), the Wanfang Database (http://www.wanfangdata.com/) (19902014.6), and reference lists of relevant articles. The terms "aripiprazole", "tourette syndrome", "tic disorders", and "tics" were combined using "and" or "or" for searching for relevant studies. The search was restricted to human studies and the language of publications was restricted to English or Chinese.

\section{Selection of studies and data extraction}

Two reviewers (Yang and Huang) independently screened the titles and abstracts of every record. Full articles were obtained when either information provided in the title or abstracts conformed to the selection criteria outlined previously, or could not be ascertained because of limited information. To include studies, data were independently extracted by each reviewer and entered into a standardized form. The data extraction form included the following contents: (1) general characteristics of studies, (2) the general characteristics of patients, (3) the diagnostic criteria, (4) sample size, (5) comparisons, (6) outcome measurements, and (7) AEs. Discrepancies were resolved by consensus.

\section{Quality assessment}

Two reviewers (Yang and Huang) independently evaluated the methodological quality of identified studies using the "risk of bias tool" under the domains of six aspects, including (1) sequence generation, (2) allocation concealment, (3) blinding, (4) incomplete outcome data, (5) selective outcome, and (6) other biases. The methodological criteria referred to the Cochrane Handbook for Systematic Reviews of Interventions, version 5.0.1 [24].

\section{Statistical methods}

Results for dichotomous outcomes are expressed as risk ratios (RR) with 95 \% CIs. Results for continuous outcomes are expressed as the mean difference (MD) (if the same scale for each trial was available) or standardized mean difference (if different scales were used). We evaluated heterogeneity among the included studies using the $\mathrm{I}^{2}$ test. We considered a value greater than $50 \%$ to indicate substantial heterogeneity and sought the potential sources of heterogeneity (clinical heterogeneity and methodological heterogeneity). Regardless of the size of heterogeneity, the random effects model was used for statistical analysis. We

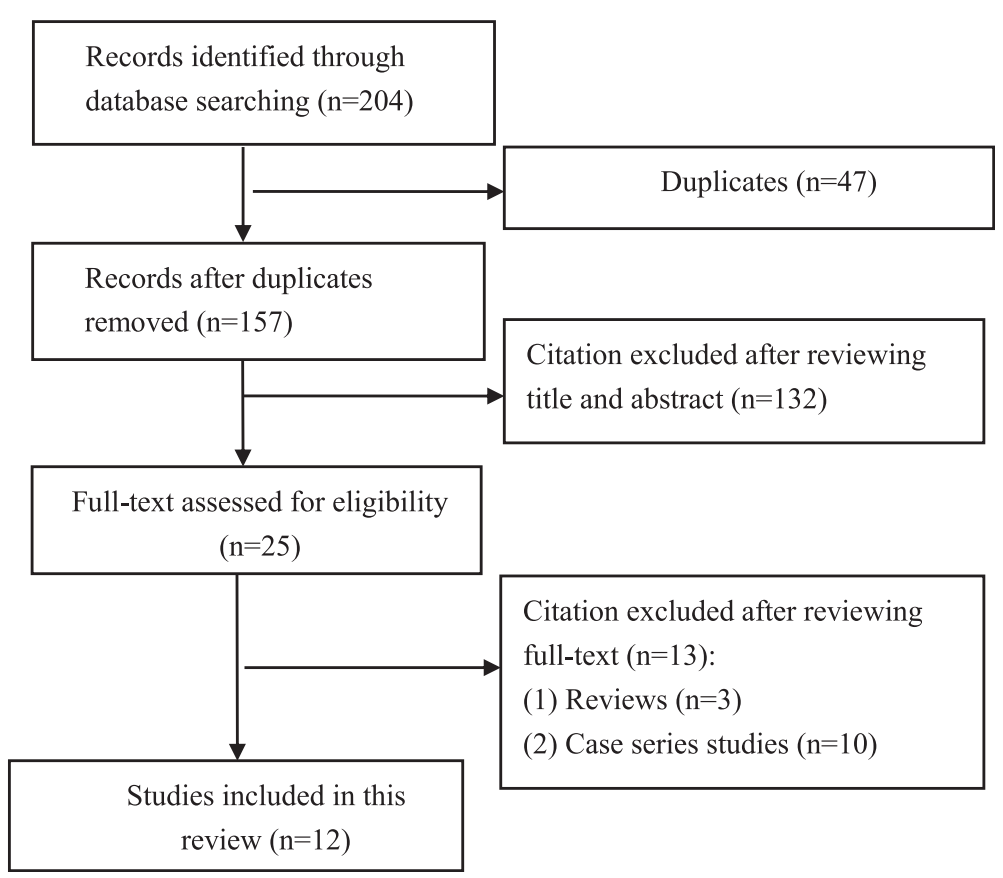

Fig 1 Flow chart of literature screening and the selection process 
Table 1 General characteristics of included studies

\begin{tabular}{|c|c|c|c|c|c|c|c|c|}
\hline \multirow[t]{2}{*}{ Study } & \multicolumn{3}{|c|}{ Characteristics of participants } & \multicolumn{2}{|l|}{ Interventions } & \multirow{2}{*}{$\begin{array}{l}\text { Treatment } \\
\text { period } \\
\text { (weeks) }\end{array}$} & \multirow{2}{*}{ Outcome measures indicators } & \multirow{2}{*}{$\begin{array}{l}\text { Diagnostic } \\
\text { criteria }\end{array}$} \\
\hline & $\begin{array}{l}\text { Age/ } \\
\text { Indication }\end{array}$ & $\begin{array}{l}\text { Sample } \\
\text { (male) }\end{array}$ & $\begin{array}{l}\text { Comparability } \\
\text { of baseline }\end{array}$ & Treatment group & Control group & & & \\
\hline \multirow{12}{*}{$\begin{array}{l}\text { Ghanizadeh } \\
2013 \text { [9] }\end{array}$} & 6-18 years; & \multirow[t]{12}{*}{$60(49)$} & \multirow[t]{12}{*}{ Comparable } & \multirow{12}{*}{$\begin{array}{l}\text { Aripiprazole (initial dose: } 1.25 \\
\mathrm{mg} / \text { day, gradually increase } \\
\text { dose, final dose: } 10-15 \mathrm{mg} / \text { day) }\end{array}$} & \multirow{12}{*}{$\begin{array}{l}\text { Risperidone (initial dose: } 0.25 \\
\mathrm{mg} \text {, gradually increase dose, } \\
\text { final dose: } 2-3 \mathrm{mg} / \mathrm{d} \text { ) }\end{array}$} & \multirow[t]{12}{*}{8} & 1. YGTSS score & DSM-IV-TR \\
\hline & \multirow[t]{11}{*}{ Tic disorder } & & & & & & Baseline $T^{*}: 30.5 \pm 13.4, C+: 31.7 \pm 10.0$ & \multirow{11}{*}{$\begin{array}{l}\text { YGTSS } \geq \\
21\end{array}$} \\
\hline & & & & & & & After treatment $T^{*}: 12.8 \pm 12, \mathrm{Ct}: 19.3 \pm 12.5$ & \\
\hline & & & & & & & 2. Motor tic severity score & \\
\hline & & & & & & & Baseline $T^{*}: 13.1 \pm 4.1, \mathrm{Ct}: 12.9 \pm 3.8$ & \\
\hline & & & & & & & After treatment $T^{*}: 4.0 \pm 4.5, \mathrm{C}+: 6.0 \pm 4.3$ & \\
\hline & & & & & & & 3. Vocal tic severity score & \\
\hline & & & & & & & Baseline $T^{*}: 4.0 \pm 4.1, C+: 6.1 \pm 5.1$ & \\
\hline & & & & & & & After treatment $T^{*}: 0.7 \pm 1.8, \mathrm{C}+3.0 \pm 3.8$ & \\
\hline & & & & & & & 4. Total tic severity scores & \\
\hline & & & & & & & Baseline $T^{*}: 16.5 \pm 6.4, C+: 19.0 \pm 7.3$ & \\
\hline & & & & & & & After treatment $T^{*}: 5.7 \pm 6.2, C+: 9.9 \pm 7.7$ & \\
\hline \multirow[t]{12}{*}{ Yoo 2013 [10] } & \multirow{12}{*}{$\begin{array}{l}\text { 6-18 years } \\
\text { Tourette } \\
\text { syndrome }\end{array}$} & \multirow[t]{12}{*}{$61(53)$} & \multirow[t]{12}{*}{ Comparable } & \multirow{12}{*}{$\begin{array}{l}\text { Aripiprazole (initial dose: } 2 \\
\text { mg/day, gradually increase } \\
\text { dose, maximum dose: } \\
20 \text { mg/day) }\end{array}$} & \multirow{12}{*}{$\begin{array}{l}\text { Placebo (initial dose: } 2 \\
\text { mg/day, gradually increase } \\
\text { dose, maximum dose: } \\
20 \text { mg/day) }\end{array}$} & \multirow[t]{12}{*}{9} & 1. Total tic severity scores & DSM-IV \\
\hline & & & & & & & Baseline $T^{*}: 28.3 \pm 5.5, C+: 29.5 \pm 5.6$ & \multirow{11}{*}{$\begin{array}{l}\text { YGTSS } \geq \\
22\end{array}$} \\
\hline & & & & & & & After treatment $T^{*}: 13.6 \pm 9.1, C+: 19.9 \pm 9.5$ & \\
\hline & & & & & & & 2. Motor tic severity score & \\
\hline & & & & & & & Baseline $T^{*}: 15.9 \pm 4.0, C+: 17.3 \pm 3.2$ & \\
\hline & & & & & & & After treatment $T^{*}: 8.6 \pm 6.1, C+: 11.9 \pm 5.5$ & \\
\hline & & & & & & & 3. Vocal tic severity score & \\
\hline & & & & & & & Baseline $T^{*}: 12.4 \pm 3.7, \mathrm{Ct}: 12.2 \pm 4.4$ & \\
\hline & & & & & & & After treatment $T^{*}: 5.0 \pm 4.6, C+: 8.0 \pm 5.5$ & \\
\hline & & & & & & & 4. Tourette's syndrome clinical global & \\
\hline & & & & & & & Baseline $T^{*}: 4.5 \pm 0.8, C+: 4.7 \pm 0.8$ & \\
\hline & & & & & & & After treatment $T^{*}: 2.8 \pm 1.4, \mathrm{C}+3.6 \pm 1.3$ & \\
\hline \multirow[t]{8}{*}{ Yoo 2011 [14] } & \multirow{8}{*}{$\begin{array}{l}\text { 6-15 years; } \\
\text { Tic disorder }\end{array}$} & \multirow[t]{8}{*}{$48(33)$} & \multirow[t]{8}{*}{ Comparable } & \multirow{8}{*}{$\begin{array}{l}\text { Aripiprazole (initial dose: } \\
5 \mathrm{mg} / \mathrm{d} \text {, increments every } \\
2 \text { weeks: } 5-10 \mathrm{mg} / \mathrm{d} \text {, maximum } \\
\text { dose: } 20 \mathrm{mg} / \mathrm{d} \text { ) }\end{array}$} & \multirow{8}{*}{$\begin{array}{l}\text { Haloperidol (initial dose } \\
0.75 \mathrm{mg} / \mathrm{d} \text { and increased in } \\
1.5-3 \mathrm{mg} / \mathrm{day} \text { increments } \\
\text { every } 2 \mathrm{weeks} \text {, maximum } \\
\text { dose: } 4.5 \mathrm{mg} / \mathrm{d}\end{array}$} & 8 & 1. Total tic severity scores: & DSM-IV \\
\hline & & & & & & & Baseline $T^{*}: 26.5 \pm 4.9, C+: 27.6 \pm 7.3$ & YGTSS $\geq$ \\
\hline & & & & & & & After treatment $T^{*}: 12.1 \pm 6.4, C+: 10.1 \pm 7.5$ & \\
\hline & & & & & & & 2. Motor tic severity scores: & \\
\hline & & & & & & & Baseline $T^{*}: 17.5 \pm 5.3, C+: 20.5 \pm 3.1$ & \\
\hline & & & & & & & After treatment $T^{*}: 8.0 \pm 4.4, C+: 8.5 \pm 6.7$ & \\
\hline & & & & & & & 3. Vocal tic severity score: & \\
\hline & & & & & & & Baseline $T^{*}: 9.0 \pm 6.7, C+: 7.1 \pm 8.3$ & \\
\hline
\end{tabular}


After treatment $T^{*} 4.5 \pm 4.6, \mathrm{C}+2.4 \pm 4.3$

Tiapride: (initial dose: $50 \mathrm{mg} / \mathrm{d}$, maximum dose $300 \mathrm{mg} / \mathrm{d}$ )

$2.5 \mathrm{mg} / \mathrm{d}$, maximum dos $2.5 \mathrm{mg} / \mathrm{d}$,
$10 \mathrm{mg} / \mathrm{d}$ )

\section{YGTSS score}

Baseline T*: $70.8 \pm 9.9$, Ct: $70.1 \pm 9.6$
60(41) Unclear syndrom $\begin{array}{ll}\text { Liu } 2010[26] & \text { 6-14 years; } \\ \text { Tic disorder }\end{array}$

Liu 2011 [27] 5-17 years; syndrom
65(57) Comparable Aripiprazole (initial dose:2.5 $\mathrm{mg}$, qd, increase dose every week:

$2.5 \mathrm{mg}$, maximum dose: $10 \mathrm{mg} / \mathrm{d})$
Tiapride (initial dose: $25 \mathrm{mg}$ bid, increase dose every week: $25 \mathrm{mg}$, maximum dose: $400 \mathrm{mg} / \mathrm{d}$.
Aripiprazole (Age $<8$ years: initial dose: $2.5 \mathrm{mg}$, qd increase dose every week: 2.5 $\mathrm{mg}$, final dose 5-15 mg/d, qd. Age $>8$ years:

initial dose $5 \mathrm{mg}$, qd, increase dose every week: $5 \mathrm{mg}$, final dose: $10-25 \mathrm{mg} / \mathrm{d}$, qd)
Tiapride (Age < 8 years: increase dose every week: 50 $\mathrm{mg}$, final dose $100-300 \mathrm{mg} / \mathrm{d}$, bid or tid. Age > 8 years: initial
dose $50 \mathrm{mg}$, bid, increase final dose: 200-500 mg/d, bid or tid)
After treatment $T^{*}: 44.5 \pm 7.9, \mathrm{C}+: 51.8 \pm 8.3$

2. Motor tic severity score

Baseline $T^{*}: 24.8 \pm 6.5, \mathrm{C}+: 23.1 \pm 5.8$

After treatment $T^{*}: 8.7 \pm 6.3, \mathrm{C}$ : $9.5 \pm 5.8$

3. Vocal tic severity score

Baseline T*: $18.8 \pm 8.4$, Ct: $18.8 \pm 8.4$

After treatment $T^{*}: 9.6 \pm 7.8, C+: 11.5 \pm 7.1$

4. Impairment score:

Baseline T*: $31.4 \pm 8.3, \mathrm{Ct}: 30.5 \pm 8.8$

After treatment $T^{*}: 18.9 \pm 7.8, C+: 22.4 \pm 7.8$

1. Author self-defined tics symptom improvement

(Rate of progress in tics symptom $\geq 30 \%$ ) T*:91\%(30/33), Ct: $84 \%(26 / 31)$

2. Decreased YGTSS score

T*: $64 \pm 23, \mathrm{C}+: 63 \pm 25$

3. Decreased motor tic severity score

T*: $68 \pm 15, \mathrm{Ct}: 61 \pm 15$

4. Decreased vocal tic severity score

T*: $68 \pm 15, \mathrm{C}+: 61 \pm 15$

5. Decrease impairment score

T*. $59+42, \mathrm{C}+\mathbf{6 3}+48$

1. YGTSS score

Baseline $T^{*}: 53.74 \pm 15.71, C+: 51.66 \pm 13.63$

After treatment $T^{*}: 24.36 \pm 16.38$,

Ct: $23.26 .1 \pm 15.31$

2. Motor tic severity score

Baseline T*: $15.93 \pm 3.22$, Ct: $15.08 \pm 2.97$

After treatment $T^{*}: 7.69 \pm 4.14, \mathrm{C}+: 7.45 \pm 3.42$

3. Vocal tic severity score

Baseline T*: $11.99+4.90$, Ct: $11.63+3.88$

After treatment $T^{*}: 4.19 \pm 4.05, C+: 3.76 .1 \pm 3.57$ 


\begin{tabular}{|c|c|c|c|c|c|c|c|c|}
\hline \multirow{4}{*}{$\begin{array}{l}\text { Cheng } 2012 \\
\text { [28] }\end{array}$} & & & & & & & & \\
\hline & $\mathrm{T}^{*}: 8.1 \pm 2.9$ & \multirow[t]{3}{*}{$62(39)$} & \multirow[t]{3}{*}{ Unclear } & \multirow{3}{*}{$\begin{array}{l}\text { Aripiprazole } \\
\text { (initial dose: } 2.5 \mathrm{mg} \text {, maximum } \\
\text { dose: } 10 \mathrm{mg} / \mathrm{d} \text { ) }\end{array}$} & \multirow{3}{*}{$\begin{array}{l}\text { Haloperidol (initial dose: } \\
0.5 \mathrm{mg} \text {, maximum dose: } \\
10 \mathrm{mg} / \mathrm{d} \text { ) }\end{array}$} & \multirow[t]{3}{*}{8} & YGTSS score & \multirow[t]{3}{*}{ CCMD-3 } \\
\hline & $C+: 7.9 \pm 3.2$ & & & & & & Baseline $T^{*}: 64.15 \pm 15.52, C+: 66.34 \pm 15.37$ & \\
\hline & Tic disorder & & & & & & After treatment $T^{*}: 17.59 \pm 15.12, \mathrm{C}+25.05 \pm 16.81$ & \\
\hline \multirow[t]{6}{*}{ Ren 2012 [29] } & \multirow{6}{*}{$\begin{array}{l}5-16 \text { years; } \\
\text { Tic disorder }\end{array}$} & \multirow[t]{6}{*}{$68(58)$} & \multirow[t]{6}{*}{ Comparable } & \multirow{6}{*}{$\begin{array}{l}\text { Aripiprazole (initial dose: } \\
2.5 \mathrm{mg} / \mathrm{d} \text {, gradually increase } \\
\text { dose, final dose: } 5-20 \mathrm{mg} / \mathrm{d} \text { ) }\end{array}$} & \multirow{6}{*}{$\begin{array}{l}\text { Haloperidol (initial dose: } \\
1 \mathrm{mg} / \mathrm{d} \text {, gradually increase } \\
\text { dose, final dose: } 2-8 \mathrm{mg} / \mathrm{d} \text { ) }\end{array}$} & \multirow[t]{6}{*}{8} & 1. YGTSS score & DSM-IV-TR \\
\hline & & & & & & & Baseline $T^{*}: 55.32 \pm 12.23, C+: 54.56 \pm 13.08$ & \multirow{5}{*}{$\begin{array}{l}\text { YGTSS } \geq \\
25\end{array}$} \\
\hline & & & & & & & After treatment $T^{*}: 21.52 \pm 18.32, \mathrm{Ct}: 20.98 \pm 16.45$ & \\
\hline & & & & & & & 2. Author self-defined tics symptom improvement & \\
\hline & & & & & & & (Rate of progress in tics symptom $\geq 30 \%$ ) & \\
\hline & & & & & & & $T^{*}: 79 \%(26 / 33), C+: 73 \%(22 / 30)$ & \\
\hline \multirow[t]{2}{*}{ Zhao 2011 [30] } & \multirow{2}{*}{$\begin{array}{l}\text { 4-15 years; } \\
\text { Tic disorder }\end{array}$} & \multirow[t]{2}{*}{ 108(72) } & \multirow[t]{2}{*}{ Comparable } & \multirow{2}{*}{$\begin{array}{l}\text { Aripiprazole (initial dose: } 5 \text { mg, } \\
\text { maintenance dose: 5-15 mg/d) }\end{array}$} & \multirow{2}{*}{$\begin{array}{l}\text { Haloperidol (initial dose:2 mg, } \\
\text { maintenance dose: 2-12 mg/d) }\end{array}$} & \multirow[t]{2}{*}{8} & CGI scale & \multirow[t]{2}{*}{ CCMD-3 } \\
\hline & & & & & & & $T^{*}: 81.3 \%(44 / 54), C+: 82.8 \%(39 / 47)$ & \\
\hline \multirow[t]{3}{*}{ Guo 2013[31] } & \multirow{3}{*}{$\begin{array}{l}4-16 \text { years; Tic } \\
\text { disorder }\end{array}$} & \multirow[t]{3}{*}{$80(55)$} & \multirow[t]{3}{*}{ Comparable } & \multirow{3}{*}{$\begin{array}{l}\text { Aripiprazole } \\
\text { (initial dose: } 2.5 \mathrm{mg} \text {, maximum } \\
\text { dose: } 12.5 \mathrm{mg} \text {, average daily } \\
\text { dose: } 7.8 \pm 1.1 \mathrm{mg} \text { ) }\end{array}$} & \multirow{3}{*}{$\begin{array}{l}\text { Haloperidol (initial dose: } 1 \mathrm{mg} \text {, } \\
\text { maximum dose: } 16 \mathrm{mg}, \\
\text { average daily dose: } 5.7 \pm 0.8 \mathrm{mg} \text { ) }\end{array}$} & \multirow[t]{3}{*}{8} & YGTSS score & ICD-10 \\
\hline & & & & & & & Baseline $T^{*}: 65.43 \pm 9.64, C+: 66.37 \pm 10.16$ & \multirow{2}{*}{$\begin{array}{l}\text { YGTSS } \geq \\
25\end{array}$} \\
\hline & & & & & & & $\begin{array}{l}\text { After treatment } T^{*}: 20.17 \pm 10.32, \mathrm{C}+: 19.87 \pm \\
9.83\end{array}$ & \\
\hline Gao 2013 [32] & $T^{*}: 11.2 \pm$ & $48(33)$ & Comparable & Aripiprazole (initial dose:2.5 & Haloperidol (initial dose: & 8 & 1.Total tic severity score & CCMD-3 \\
\hline & $\begin{array}{l}3.5 ; C+: 8.6 \pm 2.9 ; \\
\text { Tic disorder }\end{array}$ & & & $\begin{array}{l}\mathrm{mg} / \mathrm{d} \text {, increase dose every } \\
\text { week: } 2.5-5.0 \mathrm{mg} / \mathrm{d} \text {, maximum }\end{array}$ & $\begin{array}{l}1 \mathrm{mg} / \mathrm{d} \text {, increase dose every } \\
\text { week: } 2 \mathrm{mg} / \mathrm{d} \text {, maximum dose: }\end{array}$ & & Baseline $T^{*}: 26.5 \pm 4.9, C+: 27.6 \pm 7.3$ & YGTSS $\geq$ \\
\hline & & & & dose: $20 \mathrm{mg} / \mathrm{d}$ ) & $8 \mathrm{mg} / \mathrm{d}$ & & After treatment $T^{*}: 12.1 \pm 6.4, C+: 10.1 \pm 7.5$ & \\
\hline & & & & & & & 2. Motor tic severity score & \\
\hline & & & & & & & Baseline $T^{*}: 17.5 \pm 5.3, C+: 20.5 \pm 3.1$ & \\
\hline & & & & & & & After treatment $T^{*}: 8.0 \pm 4.4, C+: 8.5 \pm 6.7$ & \\
\hline & & & & & & & 3. Vocal tic severity score & \\
\hline & & & & & & & Baseline $T^{*}: 9.0 \pm 6.7, C+: 7.1 \pm 8.3$ & \\
\hline & & & & & & & After treatment $T^{*}: 4.5 \pm 4.6, C+: 2.4 \pm 4.3$ & \\
\hline Liang 2010 [33] & $4-16$ years; & $80(64)$ & Comparable & Aripiprazole (5-30 mg/d) & Haloperidol (6-16 mg/d) & 8 & YGTSS score & ICD-10 \\
\hline & syndrome & & & & & & Baseline $T^{*}: 54.95 \pm 13.98, C+: 52.97 \pm 13.54$ & YGTSS $\geq$ \\
\hline & & & & & & & After treatment $T^{*}: 35.12 \pm 13.83, \mathrm{Ct}$ : & \\
\hline & & & & & & & $19.26 \pm 14.24$ & \\
\hline
\end{tabular}

4. Impairment score

Baseline $T^{*}: 25.71 \pm 10.35, C+: 24.85 \pm 9.37$

After treatment $T^{*}: 12.45+9.95, \mathrm{Ct}: 11.96 .1+9.86$

Baseline $T^{*}: 55.32 \pm 12.23, \mathrm{C}+54.56 \pm 13.08$

2. Author self-defined tics symptom improvement

Rate of progress in tics symptom $\geq 30 \%$

CGI scale

Baseline $T^{*}: 26.5 \pm 4.9, \mathrm{C}+: 27.6 \pm 7.3$

2. Motor tic severity score

Baseline $T^{*}: 17.5 \pm 5.3, \mathrm{Ct}: 20.5+3.1$

3. Vocal tic severity score

YGTSS score

fter treatment $T^{*}: 35.12 \pm 13.83, \mathrm{C}+$

Total Tic Severity Score $=$ Motor Tic Severity score + Vocal Tic Severity score (0-50), Total Yale Global Tic Severity Scale Score = Total Tic Severity Score + Impairment score (0-100)

Decreased YGTSS score: (tics scores before treatment- tics scores after treatment) /tics scores before treatment

Rate of progress in tics symptom: (tics scores before treatment- tics scores after treatment) /tics scores before treatment

CCMD Chinese classification and diagnostic criteria of mental disorders; DSM-IV diagnostic and statistical manual of mental disorder-IV; DSM-IV-TR diagnostic and statistical manual of mental disorder-IV-Text Revision

ICD-10 international code of diseases:

*. Treatment group; t: Control group 
conducted the meta-analysis using Cochrane RevMan 5.1. We planned to assess publication bias by following the recommendations on testing for funnel plot asymmetry according to the Cochrane Handbook for Systematic Reviews on Interventions [24]. We visually assessed funnel plot asymmetry.

\section{Results}

\section{Results of the literature search}

A total of 204 articles were retrieved from searching electronic databases and reference lists (Fig. 1). After removing duplicate articles and screening titles, abstracts, and full texts, 12 studies were included in this review.

\section{Characteristics of included studies}

We included 12 studies involving 935 participants (710 were male). The sample size ranged from 48 to 195 cases. Participants were aged between 4 and 18 years. The proportion of male participants was $76 \%$ (710/935). Nine studies were conducted in mainland China, two in Korea [10, 14], and one in Iran [9]. All of the studies used pharmacological interventions. Eleven studies were positive drug controls, which were defined as the control group having an active control (i.e., test drug versus control drug, or standard treatment plus test drug versus standard treatment plus control drug). Of these 11 studies, seven studies used haloperidol as a control, three studies [25-27] used tiapride and one study [9] used risperidone. Only one study used placebo as a control [10]. For diagnostic criteria, six studies used the DSM-IV (four studies used the DSM-IV-Text Revision and two used the DSMIV), three studies used the CCMD, and three studies used the ICD-10. Seven studies used the YGTSS, one study used the CGI Scale, and two studies used self-defined criteria by the authors for measurement of outcome. The period of treatment ranged from 8 to 12 weeks. See Table 1.

\section{Quality assessment}

Twenty-five percent (3/12) of studies used an adequate method of random sequence generation $[9,29,32]$. One third (4/12) of the studies only mentioned "random allocation" without a specific description. Only one study [9] implemented adequate allocation concealment and blinding, three studies were open-label studies and did not use the method of blinding [14, 28, 33], and the remaining studies did not mention the details of blinding. Fifty percent (6/12) of studies reported loss to follow-up, and none of the studies used an intention-totreat analysis for incomplete outcome data. One study stated that the reasons for loss to follow-up did not differ between two groups [10]. Only one of the studies had registration for a protocol [10]. Therefore, whether there was selective reporting was unclear. Comparability of baseline in two of the studies was unclear [25, 28]. In other trials, there were no significant differences in the comparability of baseline between the treatment group and the control group. See Table 2.

\section{Analysis of efficacy and safety \\ Primary outcome measurements}

Evaluation of efficacy by using the YGTSS One randomized, double-blind, placebo-controlled study [10] used the total YGTSS score as the outcome measurement, and showed a significant difference in reduction of the total YGTSS score $(13.6 \pm 9.1$ vs $19.9 \pm 9.5, P<0.05)$ and vocal tic score $(5.0 \pm 4.6$ vs $8.0 \pm 5.5, P<0.05)$ between

Table 2 Quality assessment of included studies

\begin{tabular}{|c|c|c|c|c|c|c|}
\hline \multirow[t]{2}{*}{ References } & \multicolumn{6}{|l|}{ Quality assessment } \\
\hline & $\begin{array}{l}\text { Random sequence } \\
\text { generation }\end{array}$ & $\begin{array}{l}\text { Allocation } \\
\text { concealment }\end{array}$ & Blinding & $\begin{array}{l}\text { Incomplete } \\
\text { outcome data }\end{array}$ & $\begin{array}{l}\text { Selective } \\
\text { reporting }\end{array}$ & $\begin{array}{l}\text { Bias from other } \\
\text { resources }\end{array}$ \\
\hline Ghanizadeh2013 [9] & Low risk & Low risk & Low risk & Low risk & Unclear & Low risk \\
\hline Yoo 2013 [10] & Unclear & Unclear & Unclear & Low risk & Low risk & Low risk \\
\hline Yoo 2011 [14] ${ }^{a}$ & High risk & Unclear & High risk & Low risk & Unclear & Low risk \\
\hline Wang 2013 [25] & High risk & Unclear & Unclear & Low risk & Unclear & High risk \\
\hline Liu 2010 [26] & Unclear & Unclear & Unclear & Unclear & Unclear & Low risk \\
\hline Liu 2011 [27] & High risk & Unclear & Unclear & Unclear & Unclear & Low risk \\
\hline Cheng 2012 [28] & Unclear & Unclear & Unclear & Low risk & Unclear & High risk \\
\hline Ren 2012 [29] ${ }^{a}$ & Low risk & Unclear & High risk & Unclear & Unclear & Low risk \\
\hline Zhao 2011 [30] & High risk & Unclear & Unclear & Unclear & Unclear & Low risk \\
\hline Guo 2013[31] & Unclear & Unclear & Unclear & Low risk & Unclear & Low risk \\
\hline Gao 2013 [32] ${ }^{a}$ & Low risk & Unclear & High risk & Unclear & Unclear & Low risk \\
\hline Liang 2010 [33] & High risk & Unclear & Unclear & Low risk & Unclear & Low risk \\
\hline
\end{tabular}

${ }^{a}$ : they are open-label studies which did not use the method of blinding, so we justify them as high risk. Many studies did not mention the details of blinding, so we justify them as unclear 


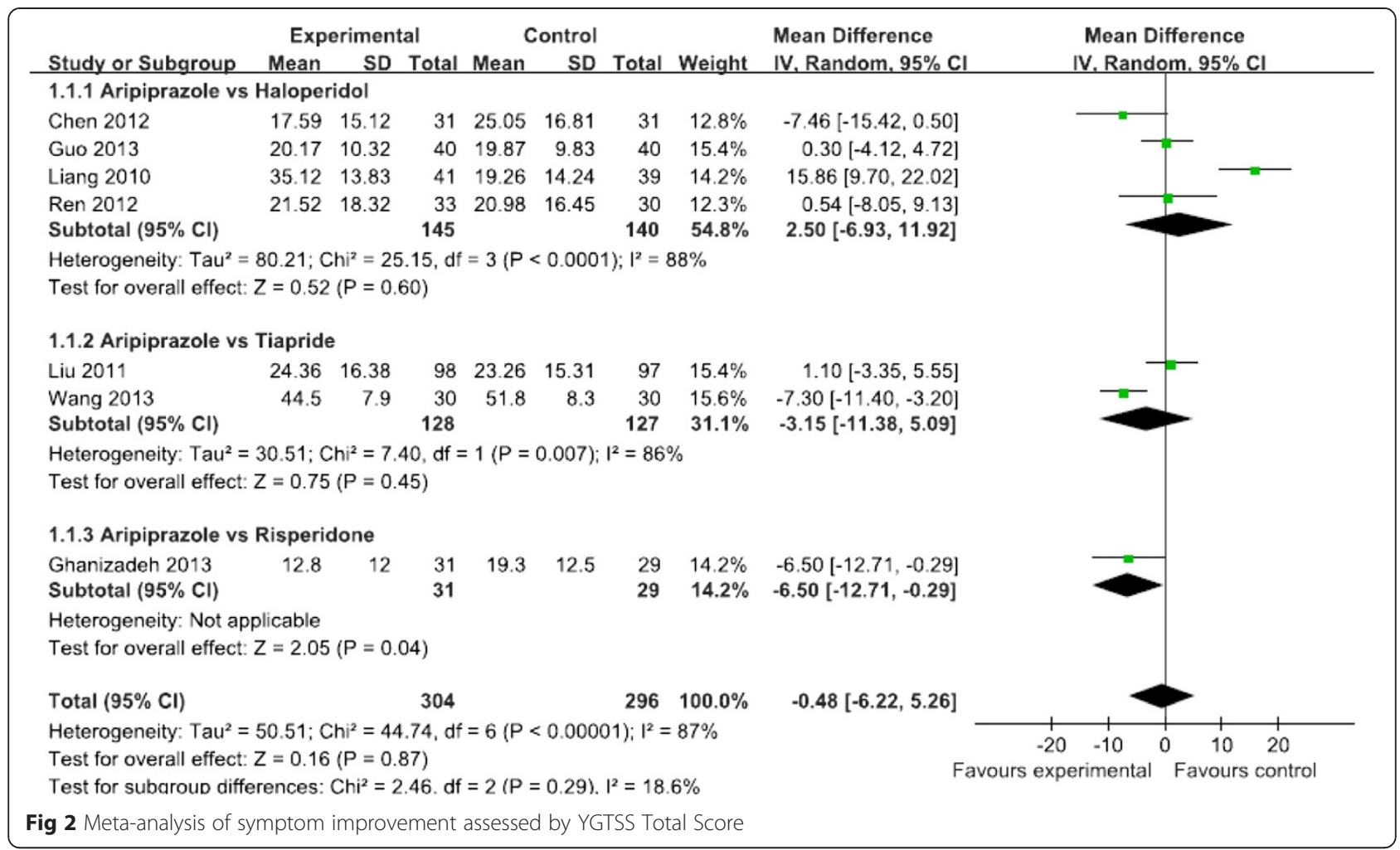

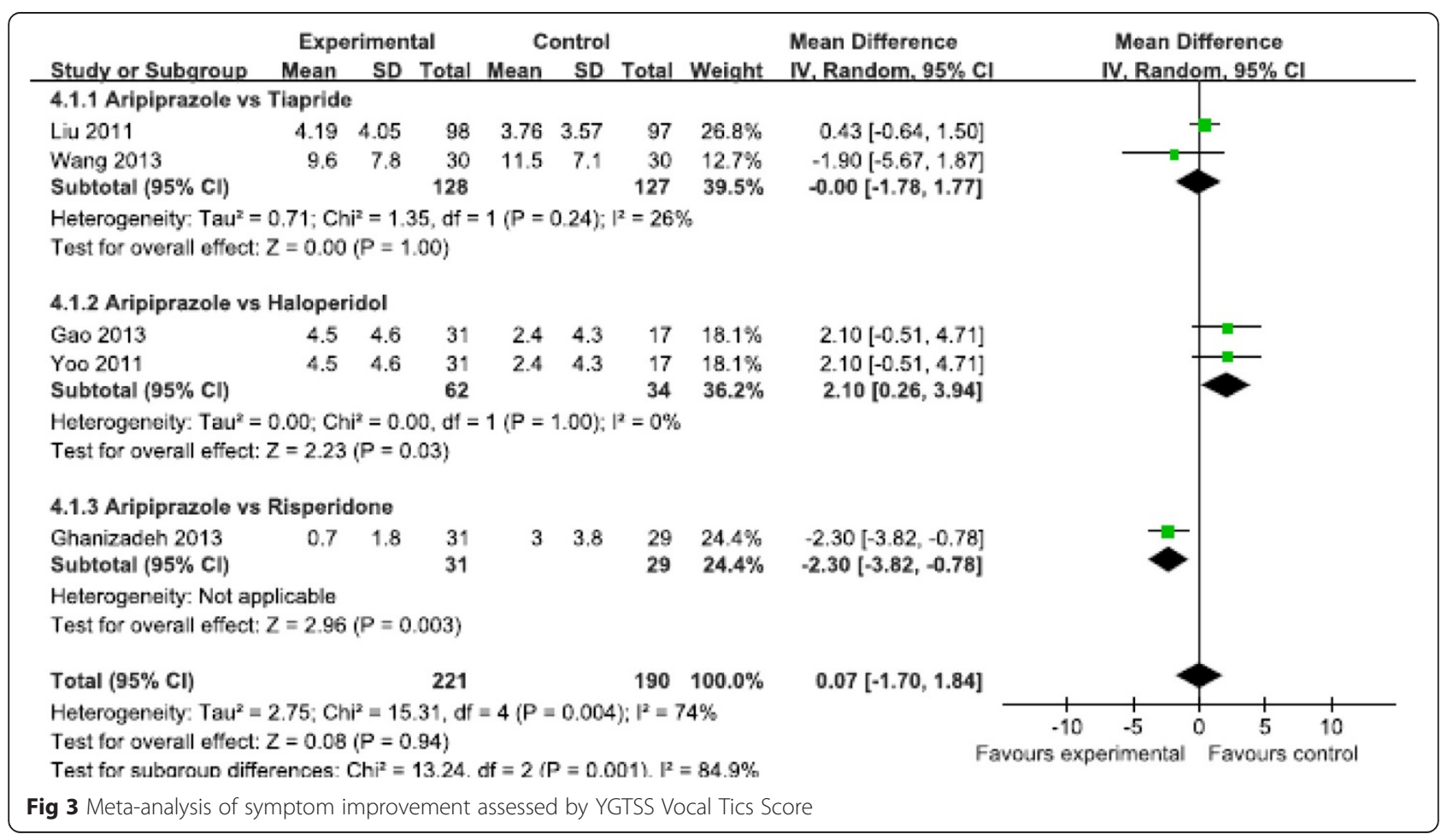




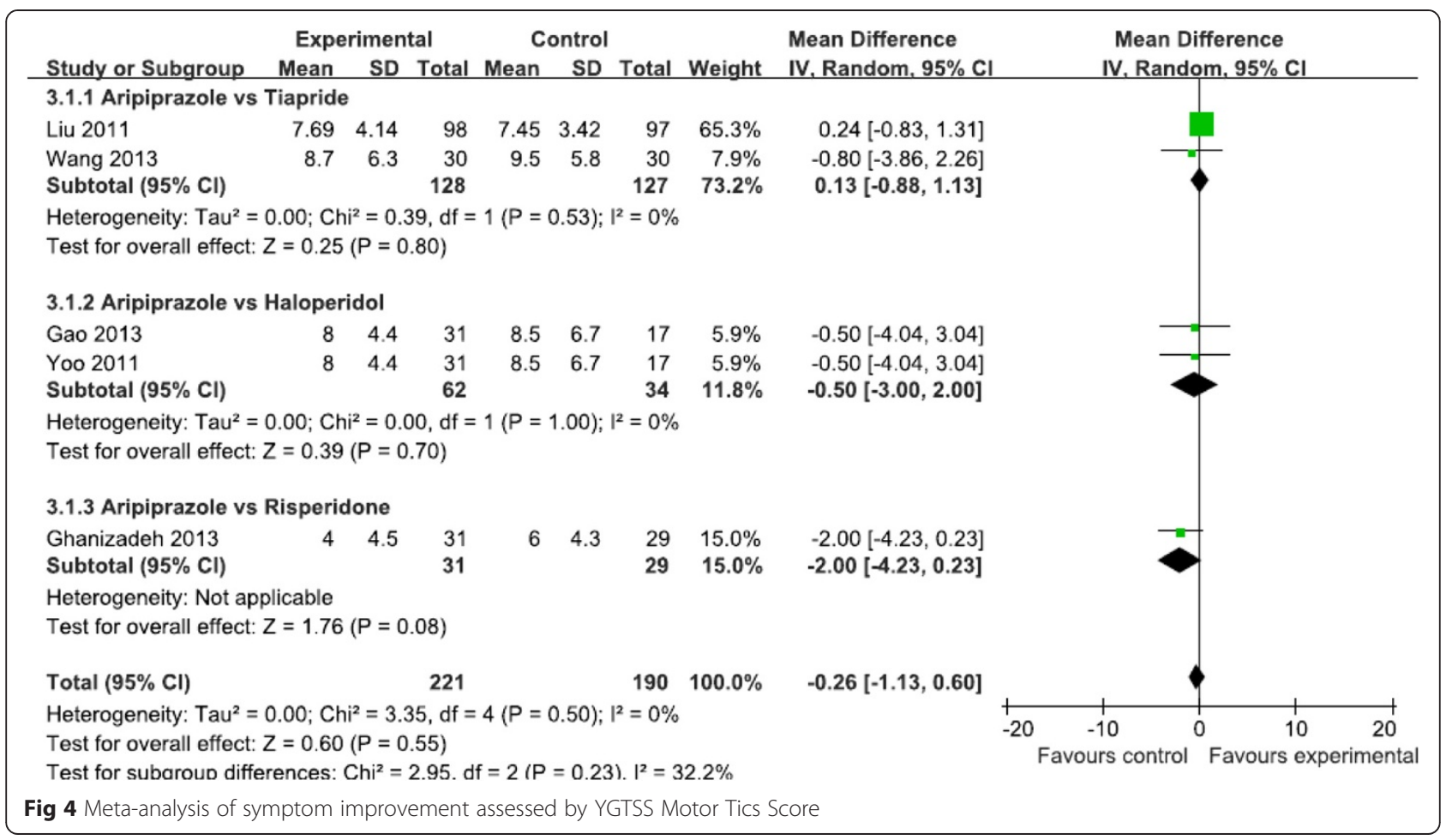

aripiprazole and placebo. There was no significant difference in reduction of the motor tic score $(8.6 \pm 6.1$ vs 11.9 $\pm 5.5, P>0.05)$. A total of seven studies ( $N=600$ patients) used the YGTSS scale as the outcome measurement, and there was no significant difference in reduction of the total YGTSS score between the aripiprazole and positive control groups $(\mathrm{MD}=-0.48,95 \% \mathrm{CI}[-6.22,5.26], P=0.87$, $\mathrm{I}^{2}=87 \%$ ) (Fig. 2) during the treatment period. Of them, meta-analysis of four of the studies $(N=285$ patients) that compared aripiprazole with haloperidol showed that there was no significant difference in reduction of the total YGTSS score between the two groups (MD $=2.50,95 \%$ CI [-6.93, 11.92], $\left.P=0.60, \mathrm{I}^{2}=88 \%\right)[28,29,31,33]$. Meta-analysis of two studies ( $N=255$ patients) that compared aripiprazole with tiapride showed that there was no significant difference in reduction of the total YGTSS score between the two groups $(\mathrm{MD}=-3.15,95 \% \mathrm{CI}$ $\left.[-11.38,5.09], P=0.45, \mathrm{I}^{2}=86 \%\right)[25,27]$. One study ( $N=60$ patients) showed that there was a significant difference in reduction of the total YGTSS score between

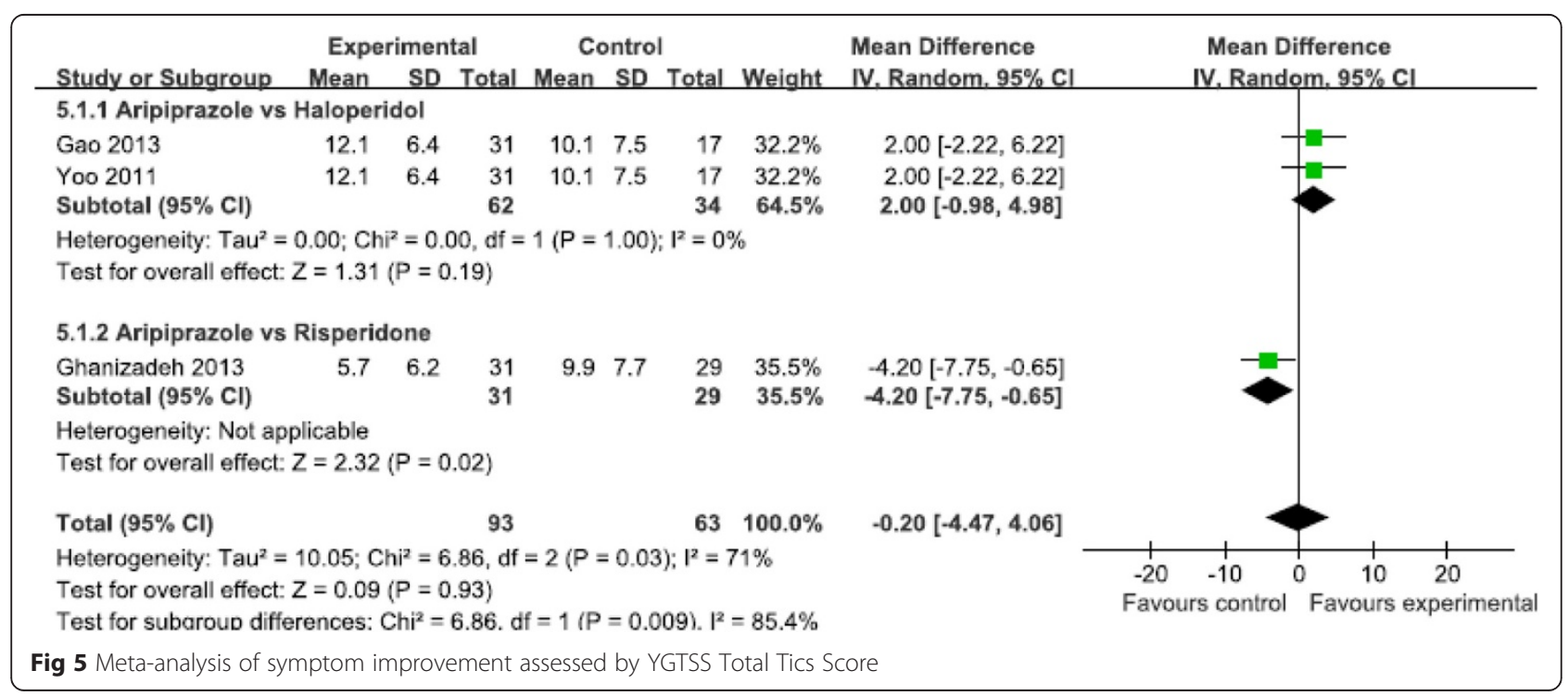




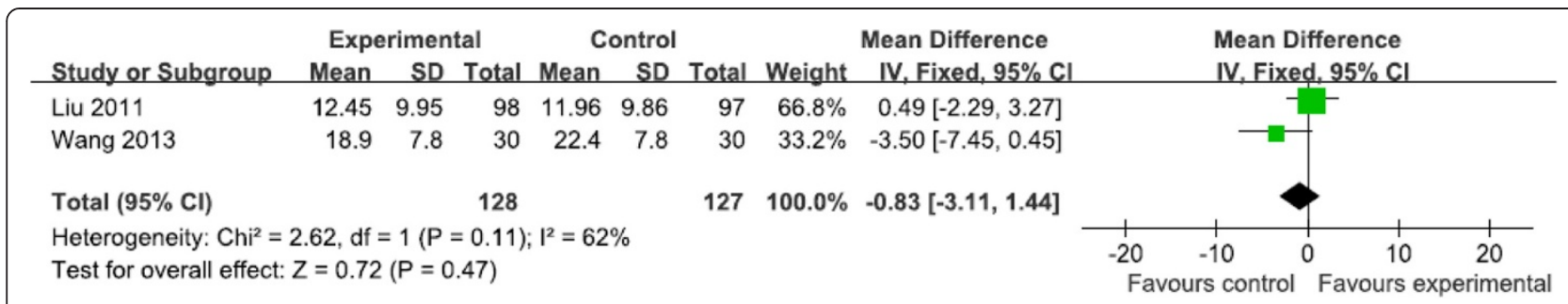

Fig 6 Meta-analysis of symptom improvement assessed by YGTSS Impairment Score

aripiprazole and risperidone $(12.8 \pm 12$ vs $19.3 \pm 12.5$, $P<0.001)$, but aripiprazole was not superior to risperidone for improving the YGTSS (MD $=-6.50$, $95 \% \mathrm{CI}$ [-12.71, -0.29], $P=0.04$ ) [9].

Five studies $(N=411$ patients) evaluated motor and vocal tics, and showed no significant difference in reduction of the vocal tics score $(\mathrm{MD}=0.07,95 \% \mathrm{CI}[-1.70$, 1.84], $P=0.94, \mathrm{I}^{2}=74 \%$ ) (Fig. 3 ) and the motor tics score $\left(\mathrm{MD}=-0.26,95 \% \mathrm{CI}[-1.13,0.60], P=0.55, \mathrm{I}^{2}=0\right)$ (Fig. 4) between the aripiprazole and positive control groups. Three studies ( $N=156$ patients) evaluated the total tic score, and showed no significant difference in reduction of the total tic score $(\mathrm{MD}=-0.2,95 \% \mathrm{CI}[-4.47$, 4.06], $P=0.93, \mathrm{I}^{2}=71 \%$ ) (Fig. 5). Two studies $(N=255$ patients) evaluated the impairment score and also showed no significant difference in reduction of the impairment score $\left(\mathrm{MD}=-0.83,95 \% \mathrm{CI}[-3.11,1.44], P=0.47, \mathrm{I}^{2}=\right.$ $62 \%)$ (Fig. 6).

Evaluation of efficacy by using the CGI Scale One study used the CGI Scale as the outcome measure, and showed no significant difference in the rate of clinical efficacy between aripiprazole and haloperidol (81.3\% [44/54] vs $82.8 \%$ [39/47], $P>0.05$ ) [30].

Evaluation of efficacy by using the Tourette Syndrome Global Scale One study used the Tourette Syndrome Global Scale as the outcome measure, and showed a significant difference in the Tourette Syndrome Global Scale between aripiprazole and placebo $(\mathrm{MD}=-0.80$, $95 \%$ CI [-1.48, -0.12], $P=0.02)$ [10].

\section{Secondary outcome measurements}

Efficacy of improvement of tic symptoms that were self-defined by authors Meta-analysis of two studies ( $N=127$ patients) used the rate of progress in tic symptoms $\geq 30 \%$ as the outcome measure. There was no significant difference between the aripiprazole and positive control groups (RR $=1.54,95 \%$ CI $\left.[0.61,3.88], P=0.36, \mathrm{I}^{2}=0\right)[29$, 31]. See Fig. 7.

AEs

All of the studies reported specific AEs, except for one study [30]. The most common AEs of aripiprazole were

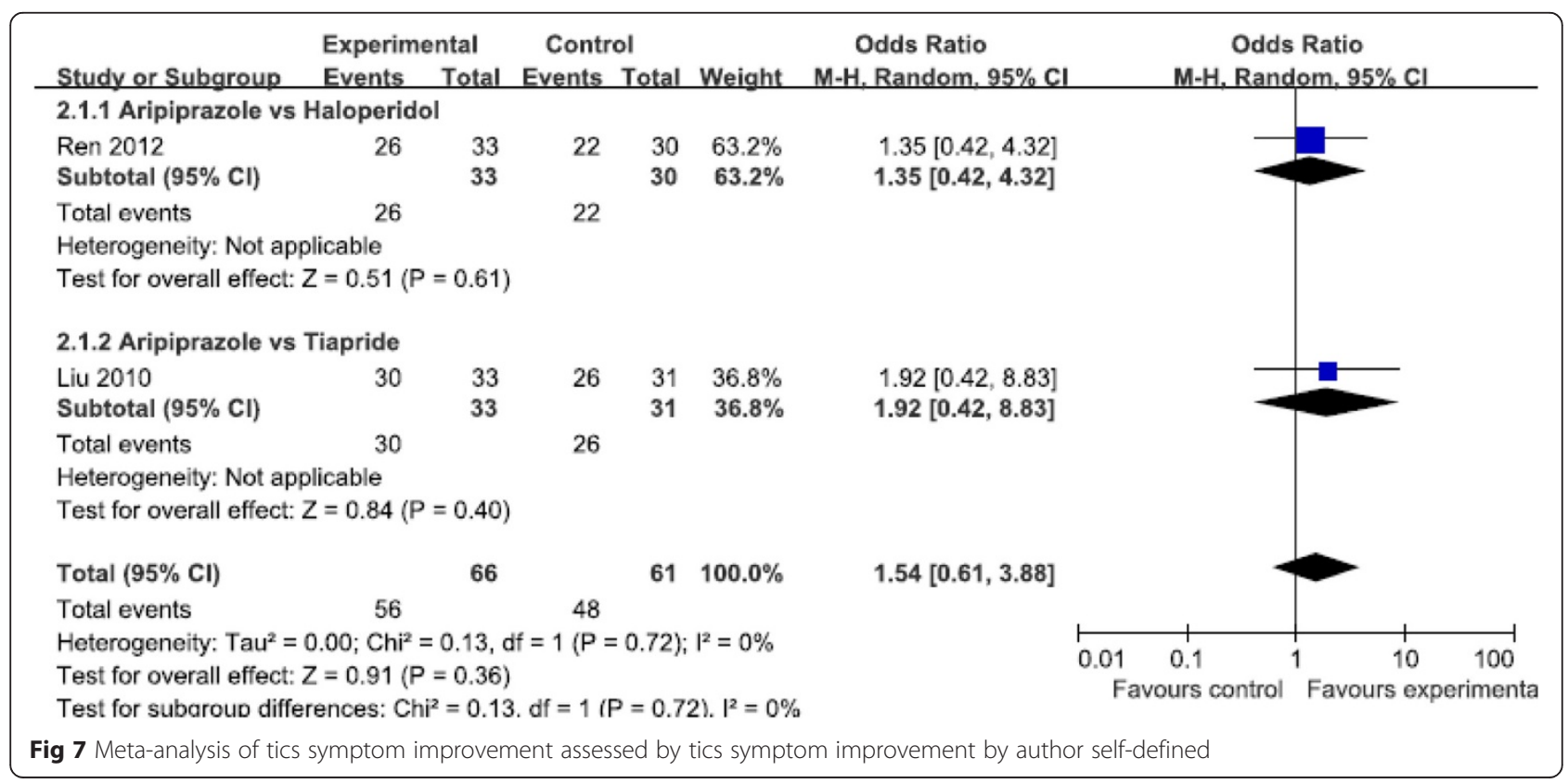


Table 3 The reported AEs of included studies

\begin{tabular}{|c|c|c|c|c|}
\hline System & Aripiprazole & Haloperidol & Tiapride & Risperidone \\
\hline \multirow{11}{*}{$\begin{array}{l}\text { Neuromuscular } \\
\text { system and } \\
\text { mental symptom }\end{array}$} & Drowsiness:5.1 \%(5/98)-58.1 \%(18/31); & Drowsiness: $6.67 \%(2 / 30)-82.4 \%(14 / 17)$ & Dizziness: $3.1 \%(3 / 97)-6.67 \%(2 / 30) ;$ & Drowsiness:17.2 \%(5/29); \\
\hline & Extrapyramidal symptoms: $6.45 \%(2 / 33)-19.4 \%(6 / 31)$ & Extrapyramidal symptoms: $40 \%(12 / 30)-43.6 \%(17 / 39) ;$ & Drowsiness: $3.23 \%(1 / 31)-5.2 \%(5 / 97)$ & Fatigue: $3.4 \%(1 / 29)$ \\
\hline & Headache: $2 \%(2 / 98)-16.1 \%(5 / 31)$; & Tremor: $19.4 \%(6 / 31)-22.5 \%(9 / 40)$ & Anxiety: $13.3 \%(4 / 30)$ & Dizziness: $3.4 \%(1 / 29) ;$ \\
\hline & Akathisia:3.33 \%(1/30)-6.3 \%(2/32); & Headache: 58.8 \%(10/17); & Sedation: $6.67 \%(2 / 30) ;$ & \multirow[t]{8}{*}{ Nausea: 3.4 \%(1/29); } \\
\hline & $\begin{array}{l}\text { Anxiety: } 2 \%(2 / 98)-6.45 \%(2 / 31) ; \text { Tremor: } \\
3.23 \%(1 / 31)-5 \%(2 / 40) ;\end{array}$ & Dizziness: $11.8 \%(2 / 17) ;$ & Akathisia: $6.67 \%(2 / 30) ;$ & \\
\hline & Fatigue: $2 \%(2 / 98)-9.7 \%(3 / 31)$ & Emotional hypersensitivity: $11.8 \%(2 / 17) ;$ & Fatigue: $3.1 \%(3 / 97)$; & \\
\hline & Dizziness: $2.44 \%(1 / 41)-6.5 \%(2 / 31)$ & $\begin{array}{l}\text { Insomnia: } 11.8 \%(2 / 17) \text {; Irritability:11.8 \%(2/17); } \\
\text { Fatigue: } 7.69 \%(3 / 39) \text {; Nightmare: } 5.9 \%(1 / 17) ;\end{array}$ & Headache: $2.1 \%(2 / 97) ;$ & \\
\hline & Insomnia: 1 \%(1/98)-3.2 \%(1/31); Sedation:12.5 \%(4/32); & & Insomnia : $2.1 \%(2 / 97)$ & \\
\hline & Slowness: $6.5 \%(2 / 31)$; Tiredness: $4.88 \%(2 / 41)$; & & & \\
\hline & $\begin{array}{l}\text { Emotional hypersensitivity: } 3.2 \%(1 / 31) \text {; Irritability: } \\
3.2 \%(1 / 31) \text {; }\end{array}$ & & & \\
\hline & Nightmare: $3.2 \%(1 / 31)$ & & & \\
\hline \multirow[t]{6}{*}{ Digestive system } & Increased appetite: $3.2 \%(1 / 31)-25.8 \%(8 / 31)$ & Nausea/vomiting: $23.5 \%(4 / 17)$ & Nausea: $3.1 \%(3 / 97)-13.3 \%(4 / 30)$ & Increased appetite: \\
\hline & $\begin{array}{l}\text { Anorexia: } 4.1 \%(4 / 98)-15 \%(6 / 40) ; \text { Nausea: } \\
2 \%(2 / 98)-18.8 \%(6 / 32) ;\end{array}$ & Nausea: $16.1 \%(5 / 31)$; & Anorexia: $3.23 \%(1 / 31)-4.1 \%(4 / 97)$; & $27.6 \%(8 / 29) ;$ \\
\hline & Nausea/vomiting:1 \%(1/98)-29 \%(9/31); & Gastrointestinal disturbances: $11.8 \%(2 / 17) ;$ & \multirow[t]{4}{*}{ Nausea/vomiting: $2.1 \%(2 / 97)$} & \multirow{4}{*}{$\begin{array}{l}\text { Abdominal pain: } \\
6.9 \%(2 / 29) ;\end{array}$} \\
\hline & $\begin{array}{l}\text { Decreased Appetite: } 12.9 \%(4 / 31) \text {; Abdominal pain } \\
9.7 \%(3 / 31) \text {; }\end{array}$ & Anorexia: $7.5 \%(3 / 40)-11.8 \%(2 / 17)$ & & \\
\hline & $\begin{array}{l}\text { Gastrointestinal disturbances: } 6.5 \%(2 / 31) \text {; Dyspepsia: } \\
3.1 \%(1 / 32) \text {; }\end{array}$ & Constipation: $6.45 \%(2 / 31)$ & & \\
\hline & Abnormal liver function: $1 \%(1 / 98)$; & Increased appetite: $5.9 \%(1 / 17)$ & & \\
\hline Ocular region & Blurred vision: $3.2 \%(1 / 31)-9.7 \%(3 / 31)$ & - & - & $\begin{array}{l}\text { Blurred vision: } \\
10.3 \%(3 / 29)\end{array}$ \\
\hline $\begin{array}{l}\text { Endocrine } \\
\text { system }\end{array}$ & Weight gain: 1 \%(1/98); Polydipsia: $3.2 \%(1 / 31)$ & - & - & - \\
\hline Urinary system & Nocturia: $3.2 \%(1 / 31)$ & Nocturia: $5.9 \%(1 / 17)$ & Nocturia: $1 \%(1 / 97)-3.23 \%(1 / 31)$ & $\begin{array}{l}\text { Diurnal Urinary } \\
\text { incontinency: } 13.8 \%(4 / 29) \text {; }\end{array}$ \\
\hline \multirow[t]{3}{*}{$\begin{array}{l}\text { Cardiovascular } \\
\text { system }\end{array}$} & Elecrocardiogram QT prolonged: 6.3 \%(2/32); & $\begin{array}{l}\text { Electrocardiographic abnormality: } \\
6.45 \%(2 / 31)-10 \%(4 / 40)\end{array}$ & \multirow[t]{3}{*}{-} & \multirow[t]{3}{*}{-} \\
\hline & Electrocardiographic abnormality: $2.5 \%(1 / 40)-6.45 \%(2 / 31)$ & Chest discomfort: $11.8 \%(2 / 17)$ & & \\
\hline & Chest discomfort: $3.2 \%(1 / 31)$ & & & \\
\hline \multirow{2}{*}{$\begin{array}{l}\text { Respiratory } \\
\text { system }\end{array}$} & Nasopharyngitis: 12.5 \%(4/32); & - & \multirow[t]{2}{*}{-} & \multirow[t]{2}{*}{-} \\
\hline & Upper respiratory tract infection: $3.1 \%(1 / 32)$ & & & \\
\hline Skin & Itches: $3.2 \%(1 / 31)$ & - & - & Itches: $10.3 \%(3 / 29)$ \\
\hline
\end{tabular}

Insomnia: $11.8 \%(2 / 17)$; Iritability:11.8 \%(2/17);

$3.1 \%(3 / 97)$

Insomnia : $2.1 \%(2 / 97)$

Emotional hypersensitivity: $3.2 \%(1 / 31)$; Irritability:

$3.2 \%(1 / 31)$

Increased appetite: $3.2 \%(1 / 31)-25.8 \%(8 / 31)$

Gastrointestinal disturbances: $11.8 \%(2 / 17)$;

Anorexia: $7.5 \%(3 / 40)-11.8 \%(2 / 17)$. 
Table 3 The reported AEs of included studies (Continued)

Others Dry mouth: $6.5 \%(2 / 31)-6.67 \%(2 / 30) ; \quad$ Dry mouth: $5 \%(2 / 40)-19.4 \%(6 / 31)$;

Dry mouth: $10 \%(3 / 30)$ :

Tiredness: $15.4 \%(6 / 39)$; Joint pain: $11.8 \%(2 / 17)$

Febrile sense: $5.9 \%(1 / 17)$;

School refusal: $5.9 \%(1 / 17)$ 
drowsiness and increased appetite. The most common AEs of haloperidol and tiapride were drowsiness, extrapyramidal symptoms, nausea, and dizziness. The most common AEs of risperidone were increased appetite, drowsiness, and diurnal urinary incontinency. The most common AEs of placebo were dizziness, akathisia and sedation. The common AEs of different drugs are shown in Table 3.

\section{Publication bias}

In this study, we visually assessed funnel plot asymmetry for the included studies that used the YGTSS for evaluation of efficacy. Figure 8 shows that the funnel plot was asymmetric.

\section{Discussion}

In this systematic review, we analyzed the efficacy and safety of aripiprazole for children with TDs, we found similar findings of efficacy among a variety of studies that compared aripiprazole and an active agent or placebo. Some studies showed that aripiprazole improved the YGTSS scores in patients with TD. Although the quality of some studies was generally poor, at least they were safe because there were no severe AEs. In general, aripiprazole was well tolerated. The most common AEs of aripiprazole were drowsiness, increased appetite, nausea, and headache. Tardive dyskinesia was observed in the typical antipsychotics (i.e., haloperidol and pimozide). To minimize such side effects, atypical antipsychotics have been prescribed for patients with TDs as alternatives. Because of a lack of long-term evaluation of outcomes, some AEs was not observed in current studies. Long-term side effects of aripiprazole should be monitored in further studies.

The quality of the included studies in our review was generally poor. The main problems are as follows. Most studies were often labeled as "random" without providing details on random sequence generation. Only one study implemented adequate allocation concealment and blinding. Successful implementation of an adequately concealed randomization sequence and blinding were not reported. Most studies used a positive drug control as a control group, with a lack of placebo controls.

Seven studies used the YGTSS as the outcome measurement, but we found large heterogeneity in the meta-analysis. The potential reasons for this large heterogeneity may be as follows. (1) Only participants with a YGTSS score $>25$ points were included in four studies $[27,29,31,33$, $35]$, the YGTSS score was $>22$ points in three studies $[10,14,32]$, and the YGTSS score was $>21$ in one study [9]. (2) There were different diagnostic criteria that were used between studies. Three studies used the DSMIV-Text Revision [9, 27, 29], three studies used the ICD10 [25, 31, 33], and one study used the CCMD-3 [28]. Patients in three of the studies $[25,27,33]$ were diagnosed with TS and the other patients had TDs. (3) There were differences in intervention and dose between studies. Four studies used haloperidol as a control, two studies used tiapride, and one study used risperidone. The initial and maximum dose varied in these included studies.

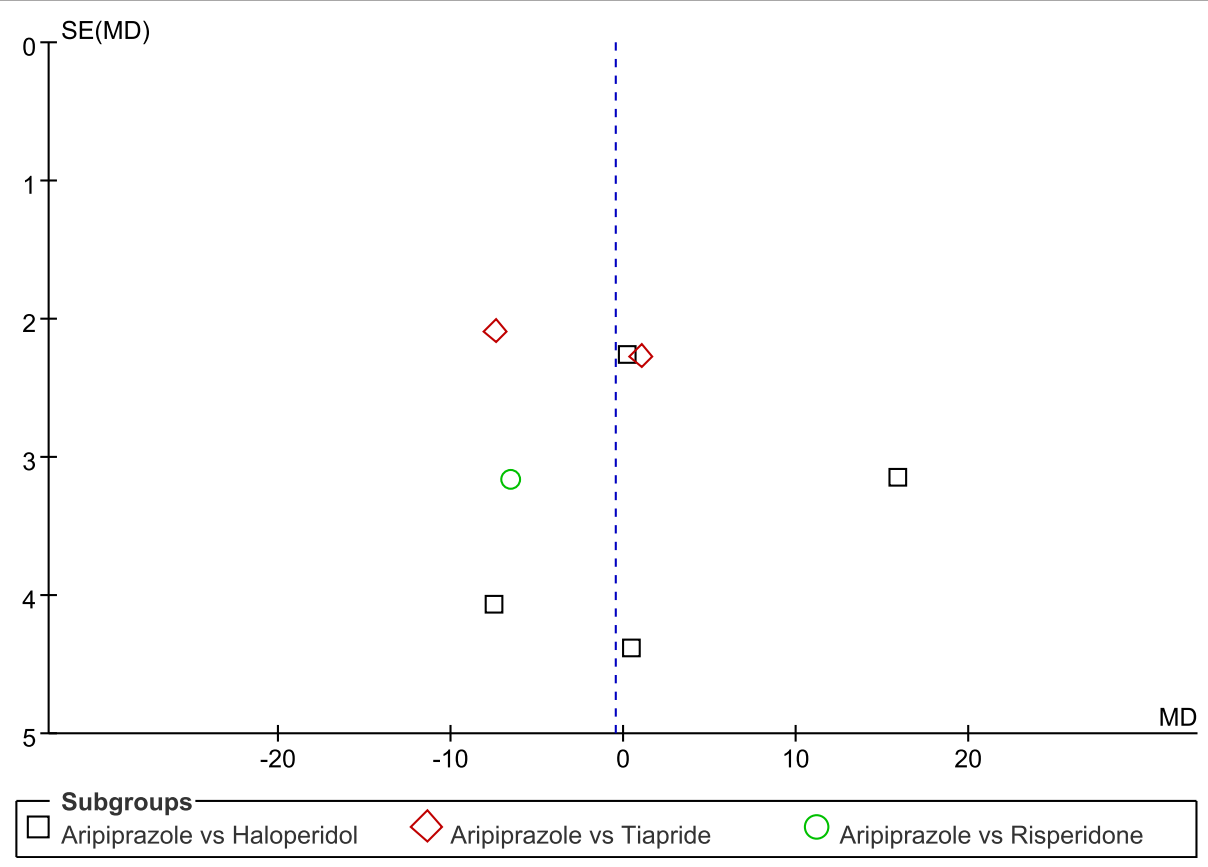

Fig 8 Funnel plot asymmetry for the included studies which using YGTSS Scale for efficacy evaluation 
TS is defined by the onset of motor and vocal tics in children, lasting more than 12 months. Although TS is the most notorious cause of chronic tics, there are types of TDs that are more common in children than in adults. According to the DSM-IV of the American Psychiatric Association, other TDs include chronic motor TD and chronic vocal TD, which are defined as having motor or phonic tics (but not both) for more than 12 months. TTD is characterized by tics (either motor and/or vocal) for a duration of less than 12 months [35]. TS is often associated with behavioral problems, such as ADHD and obsessivecompulsive disorder [35], and is more difficult to treat than other TDs. In our study, four studies evaluated the efficacy of aripiprazole for TS, and in all of them, the YGTSS score was significantly reduced from baseline to after treatment. Therefore, the efficacy of aripiprazole for TS is promising.

There are several limitations to our study. (1) Most of the included studies were conducted in a single center with a small sample (48-195 cases), and were conducted in China. Therefore, the efficacy of aripiprazole needs to be tested in other ethnicities. (2) The outcome measurements varied across different studies, which made it difficult to compare the efficacy among different studies. (3) The majority of studies used positive drugs as controls, with a lack of reasonable placebo controls. (4) There was a lack of long-term evaluation of outcomes in the included studies. AEs, such as metabolic side effects and tardive dyskinesia, were not observed in current studies. Long-term side effects of aripiprazole need to be monitored in further studies. (5) Monitoring the quality of implications and reporting of trials was difficult because of the lack of clinical trial registration, and publication bias may exist.

\section{Conclusions}

In conclusion, aripiprazole appears to be a promising therapy for children with TDs. Further well-conducted RCTs are required to confirm this issue.

\section{Competing interests}

The authors declare that they have no competing interests.

\section{Authors' contributions}

$\mathrm{CY}$ and $\mathrm{HH}$ contributed equally to this study. $\mathrm{CY}$ was involved in the design of the study, collected the data, undertook the statistical analyses and drafted the manuscript. HH was involved in the design of the study, collected the data, undertook the statistical analyses and drafted the manuscript. LZ participated in the design of the study, performed the statistical analyses, helped to interpret data and drafted the manuscript. CZ participated in the design of the study and helped to draft the manuscript. QG collected the data and helped to interpret data. All authors read and approved the final manuscript.

\section{Acknowledgments}

This study was funded by the Natural Science Foundation of China: Evidence based establishment of evaluation index system for pediatric rational drug use in China. (No. 81373381).

\section{Author details}

'Department of Pharmacy, Evidence-based Pharmacy Center, West China second hospital, Key Laboratory of Birth Defects and Related Diseases of Women and Children, Sichuan University, Chengdu, China. ${ }^{2}$ West China School of Public Health, Sichuan University, Chengdu, China. ${ }^{3}$ Department of Pediatrics, West China second hospital, Key Laboratory of Birth Defects and Related Diseases of Women and Children, Sichuan University, Chengdu, China. ${ }^{4}$ West China Second University Hospital, Sichuan University, No.20,Third Section, Renmin NanLu, Chengdu, Sichuan 610041, People's Republic of China.

Received: 8 November 2014 Accepted: 18 May 2015

Published online: 29 July 2015

\section{Reference}

1. Plessen KJ. Tic disorders and Tourette's syndrome. Eur Child Adolesc Psychiatry. 2013;22 Suppl 1:S55-60.

2. Jimenez-Shahed J. Tourette syndrome. Neurol Clin. 2009;27(3):737-55.

3. Knight T, Steeves T, Day L, Lowerison M, Jette N, Pringsheim T. Prevalence of tic disorders: a systematic review and meta-analysis. Pediatr Neurol. 2012:47(2):77-90.

4. Cavanna AE, Seri S. Tourette's syndrome. Br Med J. 2013;347:1-6.

5. Yang CS, Zhang LL, Zeng LN, Huang L, Liu YT. Topiramate for Tourette's syndrome in children: a meta-analysis. Pediatr Neurol. 2013;49(5):344-50.

6. Gilbert D, Batterson J, Sethuramamn G, Sallee F. Tic reduction with risperidone versus pimozide in a randomized double-blind cross-over trial. J Am Acad Child Adolesc Psychiatry. 2004;43:206-14.

7. Kim B, Lee B, Hwang J, Shin M, Cho S. Effectiveness and safety of risperidone for children and adolescents with chronic tic or Tourette's disorder in Korea. J Child Adolesc Psychopharmacol. 2005;15:318-24.

8. Dion Y, Annable L, Sandor P, Couinard G. Risperidone in the treatment of Tourette syndrome: a double-blind, placebo-controlled trial. J Clin Psychopharmacol. 2002;22:31-9.

9. Ghanizadeh A, Haghighi A. Aripiprazole versus risperidone for treating children and adolescents with tic disorder: a randomized double blind clinical trial. Child Psychiatry Hum Dev. 2013;45(5):596-603.

10. Yoo HK, Joung YS, Lee JS, Song DH, Lee YS, Kim JW, et al. A multicenter, randomized, double-blind, placebo-controlled study of aripiprazole in children and adolescents with Tourette's disorder. J Clin Psychiatry. 2013;74(8):772-80.

11. Ho CS, Chiu NC, Tseng CF, Huang YL. Clinical effectiveness of aripiprazole in short-term treatment of tic disorder in children and adolescents: anaturalistic study. Pediatr Neonatol. 2014;55(1):48-52.

12. Masi G, Gagliano A, Siracusano R, Berloffa S, Calarese T, llardo G, et al. Aripiprazole in children with Tourette's disorder and co-morbid attentiondeficit/hyperactivity disorder: a 12-week, open-label, preliminary study. J Child Adolesc Psychopharmacol. 2012;22(2):120-5.

13. Bowles TM, Levin GM. Aripiprazole: a new atypical antipsychotic drug. Ann Pharmacother. 2003;37:687-94.

14. Yoo HK, Lee JS, Paik KW, Choi SH, Yoon SJ, Kim JE, et al. Open-label study comparing the efficacy and tolerability of aripiprazole and haloperidol in the treatment of pediatric tic disorders. Eur Child Adolesc Psychiatry. 2011;20(3):127-35.

15. Ghanizadeh A. Systemic review of aripiprazole for the treatment of children and adolescents with tic disorders. Neurosciences (Riyadh). 2012;17(3):200-4.

16. Federowicz VJ, Fombonne E. Metabolic side effects of atypical antipsychotics in children: a literature review. J Psychopharmacol. 2005;19(5):533-50.

17. Yan WW. A review of diagnostic and statistical manual of mental disorder-III. Foreign Medical Sciences (Section of Psychiatry). 1981;6:5-9.

18. Pringsheim T, Marras C. Pimozide for tics in Tourette's syndrome. Cochrane Database Syst Rev. 2009;15(2):1-15.

19. Leckman JF, Towbin KE, Ort Sl, et al. Clinical assessment of tic disorder severity. In: Tourette's Syndrome and Tic Disorders. New York, NY: John Wiley\& Sons; 1988. p. 55-78.

20. World Health Organization. The ICD-10 Classification of Mental and Behavioral Disorders Diagnostic Criteria for Research. Geneva: WHO; 1993.

21. The Branch of Psychiatry of Chinese Medical Association. Chinese Classification and Diagnostic Criteria of Mental Disorders-3 (CCMD-3). Science and Technology Press of Shandong Province. 2001. 
22. Leckman JF, Riddle MA, Hardin MT, Ort SI, Swartz KL, Stevenson J, et al. The Yale global tic severity scale: initial testing of a clinician-rated scale of tic severity. J Am Acad Child Adolesc Psychiatry. 1989;28(4):566-73.

23. Walkup JT, Rosenberg LA, Brown J, Singer HS. The validity of instruments measuring tic severity in Tourette's syndrome. J Am Acad Child Adolesc Psychiatry. 1992:31(3):472-7.

24. Higgins JPT, Green S, editors. Cochrane Handbook for Systematic Reviews of Interventions Version 5.1.0 [updated March 2011]. The Cochrane Collaboration, 2011. Available from www.cochrane-handbook.org.

25. Wang YH, Chen ZM, Wang XS. The efficacy of aripiprazole and tiapride for Tourette disorders in children. Shandong Medical Journal. 2013;53(39):58-9.

26. Liu YY, Chen YH, Chen H, Liu ZS. A control study of aripiprazole and tiapride treatment for tic disorders in children. Chin J Contemp Pediatr. 2010;12(6):421-4.

27. Liu ZS, Chen YH, Zhong YQ, Zou LP, Wang H, Sun D, et al. A multicenter controlled study on aripirazole treatment for children with Tourette syndrome in China. Chin J Pediatr. 2011:49(8):572-6.

28. Cheng ZM, Lei QH. Comparative study of Aripiprazole and Haloperidol in the treatment of tic disorder. Medical Journal of Chinese People's Health. 2012;24(4):402-3.

29. Ren $Z B$, Jin $W D$, Wang $H Q$. A comparative study of aripiprazole and haloperidol treatment for tic disorders in children. Chinese Journal of Nervous and Mental Diseases. 2012;38(4):222-4.

30. Zhao ZL, Guo P, Guo H. The efficacy of aripiprazole and haloperidol treatment for tic disorders in children. World Health Digest Medical Periodieal. 2011;8(29):111-3.

31. Guo F, Qin X, Guo SQ, Li YL. Aripiprazole and haloperidol in the treatment of tic disorder of childhood. China Journal of Health Psychology. 2013;21(12):1767-8

32. Gao R, Zhou YD, Huang ZY, Tang JH, Lu HP. An open label control study of aripiprazole and haloperidol in the treatment of tic disorder for children. Sichuan Mental Health. 2013;26(4):300-2.

33. Liang YZ, Zhou FC, Zheng Y, Yang JH, Liu J, Wang P, et al. Comparative study of aripiprazole in the treatment of Tourette syndrome. Chinese Journal of Nervous and Mental Disease. 2010:36(2):111-2.

34. Cohen SC, Leckman JF, Bloch MH. Clinical assessment of Tourette syndrome and tic disorders. Neurosci Biobehav Rev. 2013;37(6):997-1007.

35. Waldon K, Hill J, Termine C, Balottin U, Cavanna AE. Trials of pharmacological interventions for Tourette syndrome: a systematic review. Behav Neurol. 2013;26(4):265-73.

\section{Submit your next manuscript to BioMed Central and take full advantage of:}

- Convenient online submission

- Thorough peer review

- No space constraints or color figure charges

- Immediate publication on acceptance

- Inclusion in PubMed, CAS, Scopus and Google Scholar

- Research which is freely available for redistribution 